\title{
Giardia spp EN CANINOS Y NIÑOS DE COMUNIDADES CAMPESINAS DE TRES DISTRITOS DE PUNO, PERU
}

\author{
Giardia spp in Dogs and Children in Rural Communities of Three Districts \\ of Puno, Peru
}

\author{
Olguita Pablo J. ${ }^{1}$, Amanda Chávez V. ${ }^{1,4}$, Francisco Suárez A. ${ }^{2}$, Rosa Pinedo V. ${ }^{1}$ \\ Néstor Falcón $\mathbf{P}^{3}$
}

\section{RESUMEN}

El objetivo del estudio fue determinar la prevalencia de Giardia spp en caninos y niños de comunidades campesinas de tres distritos de Puno, Perú. Se recolectaron 130 muestras fecales, tanto de niños como de canes aparentemente sanos, de ambos sexos y de diferentes edades. Se utilizaron las técnicas de Sedimentación Espontánea y de Sheather para el diagnóstico de Giardia. Se consideró una muestra como positiva si se halló al menos un resultado positivo en una de las dos técnicas. Se halló una prevalencia global de $14.6 \pm 6.1$ y $28.5 \pm 7.8 \%$ en caninos y niños, respectivamente. El sexo, edad y distrito de procedencia de los canes y niños no mostraron asociación estadística con la presencia del parásito. Los resultados positivos de Giardia spp hallados en canes y niños sugerirían un posible riesgo zoonótico.

Palabras clave: Giardia spp, Puno, sedimentación espontánea, Sheather, zoonosis

\section{Abstract}

The aim of this study was to determine the prevalence of Giardia spp in dogs and children in three rural communities in Puno, Peru. Fecal samples were collected $(n=130)$ from both children and apparently healthy dogs of both sexes and different ages. The Sheather and spontaneous sedimentation technique were used for the diagnosis of Giardia. A sample was considered positive if at least one positive result in any of the two techniques was found. The overall prevalence was $14.6 \pm 6.1$ and $28.5 \pm 7.8 \%$ in dogs and children respectively. Sex, age, and location in both children and dogs were not statistically associated with the presence of the parasite. The results of prevalence of Giardia in dogs and children suggest a possible zoonotic risk.

Key words: Giardia spp, Puno, spontaneous sedimentation, Sheather, zoonosis

\footnotetext{
${ }^{1}$ Laboratorio de Microbiología y Parasitología Veterinaria, ${ }^{2}$ Laboratorio de Medicina Veterinaria Preventiva, Facultad de Medicina Veterinaria, Universidad Nacional Mayor de San Marcos, Lima

${ }^{3}$ Facultad de Veterinaria y Zootecnia, Universidad Peruana Cayetano Heredia, Lima

${ }^{4}$ E-mail: achavezvg@gmail.com
} 
Giardia spp es un protozoario flagelado que habita en el tracto intestinal del hombre y de la mayor parte de los animales domésticos (Barr, 2000). Es un parásito cosmopolita, ampliamente distribuido en todas las latitudes y continentes, especialmente en las zonas donde hay deficientes condiciones sanitarias, de vivienda, higiene personal y nivel educativo (Rivera et al., 2002).

La transmisión es fundamentalmente fecal-oral por el consumo de aguas o alimentos contaminados con quistes o por contacto con personas o animales infectados (Cacciò et al., 2005). Se caracteriza por la producción de cuadros gastrointestinales que varían desde infección asintomática hasta la enfermedad aguda o crónica asociada con diarrea y un síndrome de malabsorción (Atías, 1994; Luján, 2006).

Esta infección parasitaria en caninos ha recibido especial atención en los últimos años, no solo por afectar la salud de los animales, sino también por presentar grandes posibilidades zoonóticas, las cuales han sido demostradas en diversas investigaciones (Molina et al., 2008). El estrecho contacto que se crea entre los perros y los pobladores de comunidades campesinas, donde ambos participan en labores de pastoreo, crea un ambiente favorable para la transmisión zoonótica.

A nivel mundial, la giardiasis es una de las causas más importantes de enfermedad diarreica aguda, especialmente en niños (Elizalde et al., 2002). Se hace necesario realizar estudios sobre la prevalencia de este parásito en caninos para evaluar el verdadero impacto que estos pueden tener sobre la salud humana. Con esta finalidad, el presente trabajo tuvo por objetivo determinar la prevalencia de Giardia spp en caninos y niños de tres distritos pertenecientes al departamento de Puno.
El estudio se llevó a cabo entre los meses de enero y abril de 2008, en los distritos de Palca y Santa Lucía, en la provincia de Lampa; así como en Ajoyani, provincia de Carabaya, ambas en el departamento de Puno. Se realizaron charlas informativas en los centros de salud de cada localidad, con la finalidad de dar a conocer las probables enfermedades zoonóticas que pueden ser transmitidas por los caninos, y, a la vez, motivar la colaboración de la comunidad en la toma de muestras. Los padres que aceptaron participar en el estudio presentaron un consentimiento escrito para la toma de muestras de heces de los niños.

Se trabajó con muestras fecales obtenidas de caninos mestizos de edad variada y de ambos sexos; asimismo, con muestras de heces de niños de hasta 12 años. No obstante, las muestras no correspondieron a niños y canes que vivían dentro de la misma casa, sino dentro de la misma comunidad.

Se proporcionaron envases de plástico a los padres de los niños y propietarios de canes para la obtención de muestras de heces. Se solicitó que las heces estuvieran libres de tierra y orina. Se recolectó una sola muestra coprológica por participante, las cuales se almacenaron en recipientes térmicos con refrigerantes para su transporte al Laboratorio de Parasitología de la Estación del Instituto Nacional de Innovación Agraria (INIA-Quimsachata). Allí, las muestras fueron conservadas en formol al $10 \%$ y transportadas al Laboratorio de Parasitología de la Facultad de Medicina Veterinaria, Universidad Nacional Mayor de San Marcos, Lima. Las muestras fueron analizadas mediante las 
Cuadro 1. Prevalencia de Giardia spp en canes y niños de comunidades campesinas de tres distritos de las provincias de Carabaya y Lampa-Puno, según técnica coproparasitológica (enero-marzo, 2008)

\begin{tabular}{|c|c|c|c|c|c|c|c|c|c|}
\hline \multirow{3}{*}{ Distrito } & \multirow{3}{*}{ N. ${ }^{o}$} & \multicolumn{4}{|c|}{ Caninos } & \multicolumn{4}{|c|}{ Niños } \\
\hline & & \multicolumn{2}{|c|}{ Sedimentación } & \multicolumn{2}{|c|}{ Sheather } & \multicolumn{2}{|c|}{ Sedimentación } & \multicolumn{2}{|c|}{ Sheather } \\
\hline & & $\mathrm{n}$ & $\%$ & $\mathrm{n}$ & $\%$ & $\mathrm{n}$ & $\%$ & $\mathrm{n}$ & $\%$ \\
\hline Ajoyani & 22 & 7 & 31.8 & 3 & 13.6 & 8 & 36.4 & 7 & 31.8 \\
\hline Palca & 22 & 4 & 18.2 & 1 & 4.5 & 3 & 13.6 & 3 & 13.6 \\
\hline Santa Lucía & 86 & 8 & 9.3 & 5 & 5.8 & 24 & 27.9 & 23 & 26.7 \\
\hline Total & 130 & 19 & $14.6 \pm 6.1$ & 9 & $6.9 \pm 4.4$ & 35 & $26.9 \pm 7.6$ & 33 & $25.4 \pm 7.5$ \\
\hline
\end{tabular}

Cuadro 2. Prevalencia de Giardia spp en canes de tres distritos de las provincias de Carabaya y Lampa en Puno, según distrito de procedencia, sexo y edad (eneromarzo, 2008)

\begin{tabular}{llccc}
\hline \multirow{2}{*}{ Variables } & & $N^{\circ}$ de muestras & \multicolumn{2}{c}{ Positivos } \\
\cline { 3 - 5 } & & 22 & $\mathrm{~N}^{\circ}$ & $\%$ \\
\hline \multirow{3}{*}{ Distritos } & Ajoyani & 22 & 7 & 31.8 \\
& Palca & 86 & 4 & 18.2 \\
& Santa Lucía & 8 & 9.3 \\
Sexo & Macho & 107 & 15 & 14.0 \\
& Hembra & 23 & 4 & 17.4 \\
\multirow{3}{*}{ Edad } & $0-6$ & 13 & 1 & 7.7 \\
(meses) & $>6-12$ & 23 & 5 & 21.7 \\
& $>12-72$ & 44 & 5 & 11.4 \\
& $>72$ & 50 & 8 & 16.0 \\
\hline \multirow{2}{*}{ Total } & & 130 & 19 & $14.6 \pm 6.1$ \\
\hline
\end{tabular}

técnicas de sedimentación espontánea (Tello, 1998) y de Sheather modificado (Urquhart et al., 2001).

El estudio fue descriptivo de corte transversal. El tamaño mínimo de muestras se determinó utilizando la fórmula para estimar una proporción (Daniel, 1996), tomando como referencia el $9.35 \%$ de prevalencia de
Giardia spp en perros del Callao (Araujo et al., 2004), y de 3.3\% en pobladores de la ribera del Lago Titicaca (Maco et al., 2002), dando como resultado 130 canes y 49 niños. Al final, se optó por un tamaño de muestra de 130 para ambos con el fin de obtener una mejor estimación. Las muestras por distritos se obtuvieron utilizando la fórmula de estratificación (Pérez, 2000). 
Cuadro 3. Prevalencia de Giardia spp en niños de tres distritos de las provincias de Carabaya y Lampa, Puno, según distrito de procedencia, sexo y edad (eneromarzo, 2008)

\begin{tabular}{llccc}
\hline \multirow{2}{*}{ Variables } & \multirow{2}{*}{$\mathrm{N}^{\circ}$ de muestras } & \multicolumn{2}{c}{ Positivos } \\
\cline { 4 - 5 } & & 22 & $\mathrm{~N} .{ }^{\circ}$ & $\%$ \\
\hline \multirow{2}{*}{ Distritos } & Ajoyani & 22 & 3 & 36.4 \\
& Palca & 86 & 26 & 13.6 \\
& Santa Lucía & 58 & 15 & 30.2 \\
Sexo & Masculino & 72 & 22 & 25.9 \\
& Femenino & 12 & 4 & 30.6 \\
Edad & $0-3$ & 64 & 19 & 33.3 \\
(años) & $>3-7$ & 54 & 14 & 29.7 \\
& $>7-12$ & 130 & 37 & 28.9 \\
\hline Total & & & & $28.5 \pm 7.8$ \\
\hline
\end{tabular}

Las variables estudiadas fueron el sexo, distrito de procedencia y grupo etario (Caninos: 0 a $6,>6$ a $12,>12$ a $72,>72$ meses; Niños: 0 a 3, >3 a 7, >7 a 12 años). La frecuencia de la parasitosis se expresó en forma porcentual con intervalos de confianza al 95\%. La asociación entre las variables de interés y la presencia de Giardia spp se analizó mediante la prueba de Chi cuadrado. Los datos se procesaron con el software SPSS v. 10.0, estableciendo la significación estadística para $\mathrm{p}<0.05$. Debido a que las muestras de niños y de canes no correspondieron a los mismos hogares, no se estableció la asociación entre parasitismo de niños y de canes. El estudio tampoco pretendió establecer una comparación estadística entre técnicas de diagnóstico.

\section{Resultados}

Mediante la prueba de sedimentación espontánea se hallaron prevalencias de 14.6 $\pm 6.1 \%$ y $26.9 \pm 7.6 \%$ en canes y niños, respectivamente; mientras que con la técnica de Sheather se encontraron prevalencias de $6.9 \pm 4.4 \%$ y $25.4 \pm 7.5 \%$ en canes y niños, respectivamente (Cuadro 1). Al considerar como resultado positivo a todo hallazgo del protozoario en al menos una de las dos técnicas diagnósticas utilizadas, las prevalencias fueron de $14.6 \pm 6.1 \%(19 / 130)$ y $28.5 \pm 7.8 \%$ (37/130), para canes y niños, respectivamente. No se encontró asociación significativa entre las variables estudiadas y la presencia del parásito en canes o en niños (Cuadros 2 y 3).

\section{Discusión}

La giardiasis es una causa común de diarrea en humanos y las infecciones crónicas afectan el crecimiento, aprendizaje y estado nutricional, particularmente en niños, siendo considerada un marcador de atraso sociocultural (Hunter y Thompson, 2005; Devera et al., 2006). Lamentablemente, a pesar de ser una enfermedad muy común en las personas, pocas veces se la considera dentro del diagnóstico diferencial.

La estrecha relación que guarda el hombre con el perro conlleva un riesgo por las infecciones parasitarias que estos pueden transmitir, representando un potencial riesgo 
de salud pública (Robertson et al., 2000). Un claro ejemplo ocurre en las comunidades campesinas, donde los perros participan en las labores de pastoreo y los niños dedican la mayor parte del día a esta actividad, especialmente en el verano, creándose así un escenario que facilita la transmisión parasitaria directa. Por esta razón, es importante evaluar la prevalencia de este parásito en niños y en los canes que están directamente relacionados con ellos, en especial en la región quechua, donde se tienen muy pocas referencias de trabajos similares (Meneses et al., 1994; Cabrera et al., 2005).

Los resultados obtenidos en niños mediante la técnica de sedimentación espontánea (26.9\%) fueron similares a reportes en niños de 1-16 años del Valle del Mantaro, Jauja (35.1\%; Marcos et al., 2002) y en niños de 0-14 años de tres comunidades del distrito de Pacaraos, provincia de Canta, Lima (31.81\%; Contreras et al., 1994), posiblemente debido a similitudes en las condiciones ambientales, sanitarias, edad, inmunidad y estado nutricional del hospedero. Sin embargo, estas prevalencias fueron superiores al $4.7 \%$ obtenido en niños de tres escuelas de nivel primaria en el distrito de Santiago de Surco, Lima (Iannacone et al., 2006). Esta menor prevalencia pudo deberse a las mejores condiciones de saneamiento ambiental, y medidas de prevención y control en la región, las cuales incluyen una mayor toma de conciencia de los pobladores sobre la importancia de la higiene personal. Por otra parte, en caninos se han reportado prevalencias de Giardia spp similares a las del presente estudio, en perros procedentes de distritos del cono sur de Lima Metropolitana (Zárate et al., 2003) y en la Provincia Constitucional del Callao (Araujo et al., 2004).

No obstante los resultados, el número de casos positivos podría haber sido mayor, pues para este parásito se recomienda el examen seriado de tres muestras tomadas en días alternos, debido a la eliminación de huevos de curso intermitente. Robertson et al. (2000) mencionan que debido a este fenómeno, la prevalencia de la infección en mascotas suele ser subestimada $y$, por lo tanto, el riesgo real en los propietarios podría ser mayor a lo que actualmente se considera. Además, aunque la técnica de sedimentación espontánea ofrece ventajas, tanto en practicidad como en sensibilidad (Larragán, 1993; Flores, 1997; Zárate et al., 2003), las pruebas de ELISA y especialmente el PCR son más sensibles (88.6 y 100\%, respectivamente) para la detección de Giardia que los métodos convencionales empleados en el presente estudio (35-50\%) (Alcaraz, 2001).

La mayor prevalencia se encontró en el distrito de Ajoyani, zona que soporta la temperatura más baja y presenta la mayor precipitación pluvial, las cuales son condiciones favorables para la permanencia de los quistes de Giardia spp en el medio ambiente; sin embargo, la prevalencia encontrada no fue estadísticamente diferente a los resultados en otros distritos.

No se logró establecer diferencia estadística entre la presentación de Giardia spp por efecto del sexo, tanto en canes y niños, tal como lo señalan diversos autores (Barr, 2000; Cordero del Campillo et al., 1999; Iannacone et al., 2006), indicando que las oportunidades de infección en ambos sexos fueron similares. Situación similar ocurrió con la edad, donde no se halló relación estadística entre la prevalencia de Giardia spp de canes y niños con la edad, en coincidencia con otros estudios (Araujo et al., 2004; Hamnes et al., 2007; Tortolero et al., 2008); aunque hay estudios que demuestran una mayor susceptibilidad en individuos jóvenes (Pérez et al., 1997; Cordero del Campillo et al., 1999; Barr, 2000).

Los resultados del presente estudio deberían alertar a las autoridades correspondientes sobre la importancia de la giardiasis como enfermedad potencialmente zoonótica presente en la población, la cual está directamente relacionada con las medidas de higiene básicas y el saneamiento ambiental. Se deberían emprender acciones encaminadas 
a mejorar estos aspectos, principalmente a través de una adecuada educación sanitaria a nivel de la comunidades y, en especial, en los centros educativos; así como la aplicación de una sencilla, económica y eficaz prueba diagnóstica como la técnica de sedimentación espontánea y un adecuado tratamiento antiparasitario en el marco del control de los parásitos intestinales.

\section{ConClusiones}

- Las prevalencias de Giardia spp en caninos y niños fueron de $14.6 \pm 6.1$ y 28.5 $\pm 7.8 \%$, respectivamente, utilizando las técnicas de sedimentación espontánea y de Sheather.

- $\quad$ El sexo, edad y distrito de procedencia de los canes y niños no mostraron asociación estadística significativa con la presencia del parásito.

- $\quad$ Los resultados positivos de Giardia spp hallados en canes y niños sugerirían un posible riesgo zoonótico.

\section{LiTERATURA CITADA}

1. Alcaraz SM. 2001. Giardia y giardiosis. [Internet], [11 setiembre 2009]. Disponible en: http://www.seimc.org/control/ revi_Para/Giardia.htm

2. Araujo W, Chávez A, Casas E, Falcón N. 2004. Prevalencia de Giardia sp. en Canis familiaris de la Provincia Constitucional del Callao. Rev Vet Inv, Perú 15(2): 145-150.

3. Atías A. 1994. Parasitología clínica. $3^{\circ}$ ed. Santiago de Chile: Publ Téc Mediterráneo. 618 p.

4. Barr SC. 2000. Infecciones entéricas protozoáricas. En: Greene CE (ed). Enfermedades infecciosas en perros y gatos. México: McGraw Hill. p 530-535.

5. Cabrera M, Verástegui M, Cabrera $R$. 2005. Prevalencia de enteroparasitosis en una comunidad altoandina de la provincia de Víctor Fajardo, Ayacucho,
Perú. Rev Gastroenterol Perú 25(2):150155.

6. Cacciò SM, Thompson RC, McLauchlin J, Smith HV. 2005. Unravelling Cryptosporidium and Giardia epidemiology. Trends Parasitol 21(9):430437.

7. Contreras O, Espinoza Y, Cruzado C. 1994. Estudio parasitológico realizado en la población infantil del distrito de Pacaraos, provincia de Lima, departamento de Lima. Rev Per Epidemiol 7(1): 44-47.

8. Cordero del Campillo M, RojoVásquez FA, Martínez AR, Sánchez MC, Hernández S, Navarrete I, Diez P, et al. 1999. Parasitología veterinaria. Madrid: McGraw Hill. 968 p.

9. Daniel D. 1996. Bioestadística: Base para el análisis de las ciencias de la salud. 5 a ed. México: Limusa. 878 p.

10. Devera R, Mago Y, Al Rumhein F. 2006. Parasitosis intestinales y condiciones socio-sanitarias en niños de una comunidad rural del Estado Bolívar, Venezuela. Rev Biomed 17: 311-313.

11. Elizalde GG, Álvaro $N$, Elizalde BG. 2002. Enfermedad diarreica aguda por Giardia lamblia. Anales Fac Med UNMSM 63(1): 25-31.

12. Flores SE. 1997. Prevalencia y características de las enteroparasitosis en diez comunidades del valle del Mantaro empleando la técnica de sedimentación. Tesis Bachiller Medicina. Lima: Univ Peruana Cayetano Heredia. 55 p.

13. Hamnes IS, Gjerde BK, Robertson LJ. 2007. A longitudinal study on the occurrence of Cryptosporidium and Giardia in dogs during their first year of life. Acta Vet Scand 49(22): 1-10.

14. Hunter PR, Thompson RC. 2005. The zoonotic transmission of Giardia and Cryptosporidium. Internat J Parasitol 35: 1181-1190.

15. Iannacone J, Benites M, Chirinos L. 2006. Prevalencia de infección por parásitos intestinales en escolares de primaria de Santiago de Surco, Lima, Perú. Parasitol Latinoam 61(1-2): 54-62. 
16. Larragán M. 1993. Comparación de los principales métodos diagnósticos para enteroparásitos. Tesis Bachiller Medicina. Lima: Univ Peruana Cayetano Heredia. 50 p.

17. Luján HD. 2006. Giardia y giardiasis. Medicina 66(1): 70-74.

18. Maco FV, Marcos RL, Terashima IA, Salmavides CF, Gotuzo HE. 2002. Distribución de la enteroparasitosis en el Altiplano peruano: Estudio en 6 comunidades rurales del departamento de Puno, Perú. Rev Gastroenterol 22: 304-309.

19. Marcos RL, Maco FV, Terashima IA, Salmavides CF, Gotuzzo HE. 2002. Prevalencia de parasitosis intestinal en niños de valle del Mantaro, Jauja, Perú. Rev Med Hered 13(3): 85-90.

20. Meneses A, Olazábal E, Serrano H, González O, Salina J. 1994. Frecuencia de giardiasis en algunas especies de animales domésticos de la provincia de Villa Clara, Cuba. Vet Méx 25: 337-340.

21. Molina N, Basualdo J, Minvielle M. 2008. Genotipo zoonótico de Giardia lamblia en Atalaya, provincia de Buenos Aires. Argentina. En: Libro de resúmenes III Congreso Latinoamericano de Zoonosis. Buenos Aires, Argentina. p 26.

22. Pérez AC, Ariza AC, Úbeda OJ, Guevara BD, De Rojas AM, Lozano $S C$. 1997. Epidemiología del parasitis- mo intestinal infantil en el valle del Guadalquivir, España. Rev Esp Salud Pública 71: 547-552.

23. Pérez C. 2000. Técnicas de muestreo estadístico. México: Alfa Omega. 603 p.

24. Rivera M, De la Parte MA, Hurtado P, Magaldi L, Collazo M. 2002. Giardiasis intestinal. Mini-revisión. Invest Clín 43(2): 119-128.

25. Robertson ID, Irwin PJ, Lymbery AJ, Thompson RCA. 2000. The role of companion animals in the emergence of parasitic zoonoses. Internat J Parasitol 30: 1369-1377.

26. Tello R, Canales M. 2000. Técnicas de diagnóstico de enfermedades causadas por enteroparásitos. Diagnóstico 39(4): 197-198.

27. Tortolero LL, Cazorla PD, Morales MP, Acosta QM. 2008. Prevalencia de enteroparásitos en perros domiciliados en la ciudad de Vela, estado Falcón, Venezuela. Rev Cient 18: 312-319.

28. Urquhart GM, Armour J, Duncan JL, Duna AM, Jennings FW. 2001. Parasitología veterinaria. $2^{\mathrm{a}}$ ed. Zaragoza: Acribia. 355 p.

29. Zárate D, Chávez A, Casas E, Falcón $N$. 2003. Prevalencia de Giardia sp. en canes de los distritos del cono sur de Lima Metropolitana. Rev Inv Vet, Perú 14(2): 134-139. 